\title{
ON CONTACT CR-SUBMANIFOLDS OF SASAKIAN MANIFOLDS
}

\author{
KOJI MATSUMOTO \\ Department of Mathematics \\ Faculty of Education \\ Yamagata University \\ Yamagata, Japan \\ (Received in June 9,1982 and is revised form in January 5, 1983)
}

ABSTRACT: Recently, K.Yano and M.Kon [5] have introduced the notion of a contact CR-submanifold of a Sasakian manifold which is closely similar to the one of a CR-submanifold of a Kaehlerian manifold defined by A.Bejancu [1].

In this paper, we shall obtain some fundamental properties of contact $C R$-submanifolds of a Sasakian manifold. Next, we shall calculate the length of the second fundamental form of a contact CR-product of a Sasakian space form (THEOREM 7.4). At last, we shall prove that a totally umbilical contact $C R$-submanifold satisfying certain conditions is totally geodesic in the ambient manifold (THEOREM 8.1). KEY WARDS AND PHRASES : Kaehlerian manifold, Sasakian space form, contact CR-product. 1980 MATHEMATICS SUBJECT CLASSIFICATION CODE : $53 B 25$.

1. INTRODUCTION.

This paper is directed to specialist readers with background in the area and appreciative of its relation of this area of study.

Let $\tilde{M}$ be a $(2 n+1)$-dimensional Sasakian manifold with structure tensors $(\phi, \xi, \eta,<,>)[4]$ and let $M$ be an $m$-dimensional Riemannian manifold isometrically immersed in $\tilde{M}$ and let <,> be the induced metric on $M$. Let $\nabla$ and $\tilde{\nabla}$ be the covariant differentiations on $M$ and $\tilde{M}$, respectively. Then the Gauss and Weingarten's formulas for $M$ are respectively given by 


$$
\begin{aligned}
& \tilde{\nabla}_{U} V=\nabla_{U} V+\sigma(U, V), \\
& \tilde{\nabla}_{U}^{\lambda}=-A_{\lambda} U+\nabla_{U}^{1} \lambda
\end{aligned}
$$

for any vector fields $U, V$ tangent to $M$ and any vector field $\lambda$ normal to $M$, where $\sigma$ denotes the second fundamental form and $\nabla^{1}$ is the normal connection. The second fundamental tensor $A_{\lambda}$ is related to $\sigma$ by

$$
<A_{\lambda} U, V>=\langle\sigma(U, V), \lambda>
$$

The mean curvature vector $H$ is defined by

$$
H=\frac{1}{m} \text { trace } \sigma
$$

The submanifold $M$ is called a minimal submanifold of $\bar{M}$ if $H=0$ and $M$ is called a totally geodesic submanifold of $\tilde{M}$ if $\sigma=0$.

For any vector field $U$ tangent to $M$, we put

$$
\phi U=P U+F U
$$

where $P U$ and $F U$ are the tangential and the normal components of $\phi U$, respectively. Then $P$ is an endomorphism of the tangent bundle $T M$ of $M$ and $F$ is a normal-bundlevalued 1 -form of $T M$.

For any vector field $\lambda$ normal to $M$, we put

$$
\phi \lambda=t \lambda+f \lambda,
$$

where $t \lambda$ and $f \lambda$ are the tangential and the normal components of $\phi \lambda$, respectively. Then $f$ is an endomorphism of the normal bundle $T^{1} M$ of $M$ and $t$ is a tangent-bundlevalued 1 -form of $T^{1} M$.

We put

$$
\xi=\xi_{1}+\xi_{2},
$$

where $\xi_{1}$ and $\xi_{2}$ are the tangential and the normal components of $\xi$, respectively. Then we can put

$$
\eta=n_{1}+n_{2}
$$

where $\eta_{1}(U)=\left\langle\xi_{1}, U\right\rangle$ and $\eta_{2}(\lambda)=\left\langle\xi_{2}, \lambda\right\rangle$ for any vector field $U$ tangent to $M$ and any vector field $\lambda$ normal to $M$.

By virtue of $(1.5),(1.6),(1.7)$ and $(1.8)$, we get 


$$
\begin{aligned}
& P^{2} U+t F U=-U+\eta_{1}(U) \xi_{1}, \\
& F P U+f F U=\eta_{1}(U) \xi_{2}, \\
& \eta_{2}(\lambda) \xi_{1}=P t \lambda+t f \lambda, \\
& F t \lambda+f^{2} \lambda=-\lambda+\eta_{2}(\lambda) \xi_{2}
\end{aligned}
$$

for any vector field $U$ tangent to $M$ and any vector field $\lambda$ normal to $M$.

Let $\bar{M}(k)$ be a Sasakian space form with constant $\phi$-holomorphic sectional curvature $k$. Then the curvature tensor $\tilde{R}$ of $\tilde{M}(k)$ is given by

$$
\begin{aligned}
\tilde{R}(X, Y) Z & =\frac{k+3}{4}\{<Y, Z>X-<X, Z>Y\}+\frac{k-1}{4}\{n(X)<Y, Z>\xi-n(Y)<X, Z>\xi \\
& +n(Y) n(Z) X-n(X) n(Z) Y-<\phi Y, Z>\phi X+<\phi X, Z>\phi Y+2<\phi X, Y>\phi Z\}
\end{aligned}
$$

for any vector fields $X, Y$ and $Z$ in $\tilde{M}(k)[3]$.

For the second fundamental form $\sigma$, we define the covariant differentiation $\bar{\nabla}$ with respect to the connection on $T M \oplus T^{1} M$ by

$$
\left(\bar{\nabla}_{U} \sigma\right)(V, W)=\nabla_{U}^{1}(\sigma(V, \dot{W}))-\sigma\left(\nabla_{U} V, W\right)-\sigma\left(V, \nabla_{U} W\right)
$$

for any vector fields $U, V$ and $W$ tangent to $M$. We denote $R$ the curvature tensor associated with $\nabla$. Then the equations of Gauss and Codazzi are respectively given by

$$
\begin{aligned}
& \tilde{R}(U, V ; W, Z)=R(U, V ; W, Z)+\langle\sigma(U, W), \sigma(V, Z)\rangle-\langle\sigma(U, Z), \sigma(V, W)\rangle, \\
& (\tilde{R}(U, V) W)^{1}=\left(\bar{\nabla}_{U} \sigma\right)(V, W)-\left(\bar{\nabla}_{V} \sigma\right)(U, W)
\end{aligned}
$$

for any vector fields $U, V, W$ and $Z$ tangent to $M$, where $\tilde{R}(U, V ; W, Z)=\langle\tilde{R}(U, V) W, Z\rangle$ and $(\tilde{R}(U, V) W)^{\perp}$ denotes the normal component of $\tilde{R}(U, V) W$.

\section{CONTACT CR-SUBMANIFOLDS OF A SASAKIAN MANIFOLD.}

DEFINITION 2.1: A submanifold $M$ of a Sasakian manifold $\tilde{M}$ with structure tensors $(\phi, \xi, \eta,<,>)$ is called a contact lik-sunmanifeld if there is a differentiable distribution $D: x \longrightarrow D_{x} \subseteq T_{x}$ on $M$ satisfying the following conditions:

(i) $\xi \varepsilon D$,

(ii) $\quad \phi D_{x} \subset T_{x} M$ for each $x$ in $M$,

(iii) the complementary orthogonal distribution $D^{1}: x \longrightarrow D_{x}^{1} \subset T_{x} M$ satisfies $\phi D_{x}^{1} \subseteq T_{x}^{1} M$ for each point $x$ in $M$.

Let $M$ be a contact $C R$-submanifold of a Sasakian manifold $\tilde{M}$. Then $\xi \varepsilon D \subset T M$, 
so, the equations $(1.9),(1.10),(1.11)$ and $(1.12)$ can be written as

$$
\begin{aligned}
& P^{2} U+t F U=-U+n(U) \xi, \\
& F P U+f F U=0, \\
& P t \lambda+t f \lambda=0, \\
& F t \lambda+f^{2} \lambda=-\lambda
\end{aligned}
$$

for any vector field $U$ tangent to $M$ and any vector field $\lambda$ normal to $M$, respectively. By virtue of $(1.5)$, we have

PROPOSITION 2.1: In a contact $C R$-submanifold $M$ of a Sasakian manifold $\tilde{M}$, in order to a vector field $U$ tangent to $M$ belong to $D$ it is necessary and sufficient that $F U=0$.

Taking account of (2.1) and PROPOSITION 2.1, we have

$$
P^{2} X=-X+n(X) \xi
$$

for any $X$ in $D$ and we have from (1.6)

$$
P \xi=0 \text {. }
$$

Furthermore, we obtain

$$
\langle P X, P Y\rangle=\langle\phi X, \phi Y\rangle=\langle X, Y\rangle-\eta(X) \eta(Y)
$$

for any $X$ and $Y$ in $D$. Thus we have

PROPOSITION 2.2: In a contact $C R$-submanifold $M$ of a Sasakian manifold $\tilde{M}$, the distribution $D$ has an almost contact metric structure $(P, \xi, \eta,<,>)$ and hence $\operatorname{dim} D_{x}=$ odd.

We denote by $\Pi$ the complementary orthogonal subbundle of $\phi D^{\perp}$ in $T^{1} M$. Then we have

$$
T^{1} M=\phi D^{\perp} \oplus \Pi, \quad \phi D^{1} \perp \Pi
$$

Thus we have

PROPOSITION 2.3: For a contact $C R$-submanifold $M$ of a Sasakian manifold $\tilde{M}$, the subbundle $\Pi$ has an almost complex structure $f$ and hence $\operatorname{dim} \Pi_{x}=$ even.

\section{BASIC PROPERTIES.}

Let $M$ be a contact $C R$-submanifold of a Sasakian manifold $M$. Then we have 


$$
\phi\left(\nabla_{J} Z+\sigma(U, Z)\right)=-A_{\phi Z} U+\nabla_{U}(\phi Z)-<U, Z>\xi
$$

for any vector field $U$ tangent to $M$ and $Z$ in $D^{1}$. From (3.1), we get

$$
<\nabla_{U} Z, \phi X>=\left\langle A_{\phi Z} U, X>+n(X)<U, Z>\right.
$$

for any vector field $U$ tangent to $M, X$ in $D$ and $Z$ in $D^{1}$. In (3.2), if we put $X=\phi X$, then (3.2) means

$$
\left\langle\nabla_{U} Z, X>=\left\langle\phi A_{\phi Z} U, X>+n(X)<\nabla_{U} Z, \xi>\right.\right.
$$

for any vector field $U$ tangent to $M, X$ in $D$ and $Z$ in $D^{\perp}$. By virtue of (3.2), we obtain

$$
\left.\left\langle A_{\phi Z^{X}}, \xi\right\rangle=0 \quad \text { (or equivalently }<\sigma(X, \xi), \phi Z>=0\right)
$$

for any $X$ in $D$ and $Z$ in $D^{1}$.

On the otner hand, we have

$$
\langle\sigma(X, \xi), \lambda\rangle=\left\langle\tilde{\nabla}_{X^{\prime}}-\nabla_{X} \xi, \lambda\right\rangle=\langle\phi X, \lambda\rangle=0
$$

for any $X$ in $D$ and $\lambda$ in $\Pi$. Thus we have from (3.4) and the above equation $\sigma(X, \xi)=0$ for any $X$ in $D$. So, we have from (1.7) and the last equation

$$
\nabla_{X^{\xi}}=P X
$$

for any $X$ in $D$. Thus we have

PROPOSITION 3.1: In a contact $C R$-submanifold $M$ of a Sasakian manifold $\tilde{M}$, the distribution $D$ has a $K$-contact metric structure $(P, \xi, \eta,<,>)$.

In (3.1), if we put $U=W \varepsilon D^{1}$, then the equation (3.1) can be written as

$$
\phi\left(\nabla_{W} Z+\sigma(Z, W)\right)=-A_{\phi Z} W+\nabla_{W}^{1} \phi Z-<W, Z>\xi
$$

from which

$$
\phi([Z, W])=A_{\phi Z} W-A_{\phi W} Z+\nabla_{Z}^{1} \phi W-\nabla_{W}^{1} \phi Z,
$$

where $[Z, W]=\nabla_{Z} W-\nabla_{W} Z$.

LEMMA 3.2: In a contact $C R$-submanifold $M$ of a Sasakian manifold $\tilde{M}$, we have

$$
\nabla_{W}^{1} \phi Z-\nabla_{Z}^{1} \Phi W \varepsilon \phi D^{1}
$$

for any $Z$ and $W$ in $D^{\perp}$. 
PROOF: For any $Z, W$ in $D^{\perp}$ and $\lambda$ in $\Pi$, we obtain

$$
\begin{aligned}
& \left\langle\nabla_{W}^{1} \phi Z-\nabla_{Z}^{1} \phi W, \lambda\right\rangle=\left\langle\tilde{\nabla}_{W} \phi Z-\tilde{\nabla}_{Z} \phi W, \lambda\right\rangle=\left\langle\phi\left(\tilde{\nabla}_{W} Z-\tilde{\nabla}_{Z} W\right)+\left(\tilde{\nabla}_{W} \phi\right) Z-\left(\tilde{\nabla}_{Z} \phi\right) W, \lambda\right\rangle \\
& =\left\langle\phi\left(\nabla_{W} Z-\nabla_{Z} W\right), \lambda\right\rangle=\left\langle\nabla_{Z} W-\nabla_{W} Z, \phi \lambda\right\rangle=0 .
\end{aligned}
$$

On the other hand, we can easily have

$$
A_{\phi Z} W=A_{\phi W} Z
$$

for any $Z$ and $W$ in $D^{1}$. In fact, for any vector field $U$ tangent to $M$ and $Z$ and $W$ in $D^{1}$, we have from (3.1)

$$
\begin{aligned}
& \left\langle\phi\left(\nabla_{U} Z+\sigma(U, Z)\right), W\right\rangle=\left\langle\phi \nabla_{U} Z, W\right\rangle+\langle\phi \sigma(U, Z), W\rangle=-\langle\sigma(U, Z), \phi W\rangle \\
& =-\left\langle A_{\phi W} Z, U>=-\left\langle A_{\phi Z} W, U\right\rangle,\right.
\end{aligned}
$$

from which, we have (3.8).

By virtue of (3.6) and (3.8) and LEMMA 3.2, we have

PROPOSITION 3.3: In a contact $C R$-submanifold $M$ of a Sasakian manifold $\tilde{M}$, the distribution $D^{1}$ is integrable.

For any $X$ in $D$ and $\lambda$ in $\Pi$, we have

$$
A_{\lambda} \phi X=-A_{\phi \lambda} X
$$

Next, we assume that the distribution $D$ is integrable. Then for any $X$ and $Y$ in $D, \phi[X, Y]$ is an element of $D$, that is, $\phi[X, Y] \varepsilon T M$. Since we have

$$
\phi[X, Y]=\phi\left(\tilde{\nabla}_{X} Y-\tilde{\nabla}_{Y} X\right)=\left\{\nabla_{X} \phi Y-\nabla_{Y} \phi X+\eta(X) Y-\eta(Y) X\right\}+\{\sigma(X, \phi Y)-\sigma(\phi X, Y)\},
$$

we get $\sigma(X, \phi Y)=\sigma(\phi X, Y)$. From which we obtain

$$
\langle\sigma(X, \phi Y), \phi Z>=\langle\sigma(\phi X, Y), \phi Z>
$$

for any $X$ and $Y$ in $D$ and $Z$ in $D^{1}$.

Conversely, if (3.10) is satisfied, we can easily show that the distribution $D$ is integrable. Thus we have

PROPOSITION 3.4: In a contact $C R$-submanifold $M$ of a Sasakian manifold $\tilde{M}$, the distribution $D$ is integrable if and only if the equation (3.10) is satisfied.

Next, we can prove

PROPOSITION 3.5: In a contact $C R$-submanifold $M$ of a Sasakian manifold $\tilde{M}$, the vector field $\xi$ is parallel along $D^{1}$.

PROOF: For any $Z$ in $D^{\perp}$, we have 


$$
\phi Z=\nabla_{Z} \xi+\sigma(Z, \xi)
$$

Since the vector field $Z$ is an element of $D^{1}, \phi Z$ is in $T^{1} M$. Thus we have from the above equation $\nabla_{Z} \xi=0$, that is, the vector field $\xi$ is parallel along any vector field in $D^{1}$.

4. SOME COVARIANT DIFFERENTIATIONS.

DEFINITION 4.1: In a contact $C R$-submanifold $M$ of a Sasakian manifold $\tilde{M}$, we define

$$
\begin{aligned}
& \left(\bar{\nabla}_{U} P\right) V=\nabla_{U}(P V)-P \nabla_{U} V, \\
& \left(\bar{\nabla}_{U}^{F}\right) V=\nabla_{U}^{1}(F V)-F \nabla_{U}^{V}, \\
& \left(\bar{\nabla}_{U} t\right) \lambda=\nabla_{U}(t \lambda)-t \nabla_{U}^{1} \lambda, \\
& \left(\bar{\nabla}_{U} f\right) \lambda=\nabla_{U}^{1}(f \lambda)-f \nabla_{U}^{1} \lambda,
\end{aligned}
$$

for any vector fields $U$ and $V$ tangent to $M$ and any vector field $\lambda$ normal to $M$ [2].

DEFINITION 4.2: The endomorphism $P$ (resp. the endomorphism $f$, the 1-forms $F$ and $t$ ) is parallel if $\bar{\nabla} P=0$ (resp. $\bar{\nabla} f=0, \bar{\nabla} F=0$ and $\bar{\nabla} t=0$ ).

By virtue of $(1.5)$ and $(1.6)$, we can prove

PROPOSITION 4.1: For the covariant differentiations defined in DEFINITION 4.1, we have

$$
\begin{aligned}
& \left(\bar{\nabla}_{U} P\right) V=\langle U, V\rangle+n(V) U+t \sigma(U, V)+A_{F V} U, \\
& \left(\bar{\nabla}_{U} F\right) V=f \sigma(U, V)-\sigma(U, P V), \\
& \left(\bar{\nabla}_{U} t\right) \lambda=A_{f \lambda} U-P A_{\lambda} U, \\
& \left(\nabla_{U} f\right) \lambda=-F A_{\lambda} U-\sigma(U, t \lambda)
\end{aligned}
$$

tor any vector fields $U$ and $V$ tangent to $M$ and any vector field $\lambda$ normal to $M$.

By virtue of (4.5), we get

$$
\left(\bar{\nabla}_{X} P\right) Y=-<X, Y>\xi+n(Y) X+t \sigma(X, Y)
$$

for any $X$ and $Y$ in $D$. Thus we have

PROPOSITION 4.2: In a contact $C R$-submanifold $M$ of a Sasakian manifold $\tilde{M}$, the structure $(P, \xi, \eta,<,>)$ is Sasakian if and only if $\sigma(X, Y)$ is in $\Pi$ for any $X$ and $Y$ in $D$. 
COROLLARY 4.3: In a contact $C R$-submanifold $M$ of a Sasakian manifold $\tilde{M}$, if dim $D_{x}^{1}=0$, then the submanifold $M$ is a Sasakian submanifold.

Next, we assume that the endomorphism $P$ is paralle1. Then we have from (4.9)

$$
t \sigma(X, Y)=\langle X, Y>\xi-n(Y) X
$$

for any $X$ and $Y$ in $D$. By virtue of $\sigma(X, \xi)=0$ and $(4.10)$, we nave $X=\alpha \xi$ for any $X$ in $D$, where $\alpha$ is a certain scalar field on $D$. Thus we have

PROPOSITION 4.4: In a contact $C R$-submanifold $M$ of a Sasakian manifold $\tilde{M}$, if the endomorphism $P$ is parallel, then $\operatorname{dim} D_{x}=1$.

5. THE DISTRIBUTION $D^{1}$.

In a contact $C R$-submanifold $M$ of a Sasakian manifold $\tilde{M}$, we assume that the leaf $M^{1}$ of $D^{1}$ is totally geodesic in $M$, that is, $\nabla_{Z} W$ is in $D^{1}$ for any $Z$ and $W$ in $D^{1}$. This means

$$
<Z_{Z} W, \phi X>0
$$

for any $X$ in $D$ and $Z$ and $W$ in $D^{1}$. By virtue of PROPOSITION 3.4 and (5.1), we have

$$
<\sigma(X, Z)-n(X) \phi Z, \phi W>0
$$

for any $X$ in $D$ and $Z$ and $W$ in $D^{1}$.

Conversely, if the equation (5.2) is satisfied, then it is clear that the leaf $M^{1}$ of $D^{1}$ is totally geodesic in $M$. Thus we have

PROPOSITION 5.1: In a contact $C R$-submanifold $M$ of a Sasakian manifold $\tilde{M}$, the leaf $M^{1}$ of $D^{1}$ is totally geodesic in $M$ if and only if the equation (5.2) is satisfied.

Next, let us prove

THEOREM 5.2: In a contact $C R$-submanifold $M$ of a Sasakian manifold $\tilde{M}$, we assume that the leaf $M^{1}$ of $D^{1}$ is totally geodesic in $M$. If the endomorphism $P$ satisfies

$$
\left.\left(\bar{\nabla}_{U} P\right) V=n(V) U-<U, \backslash\right\rangle \xi
$$

for any vector fields $U$ and $V$ tangent to $M$, then $\operatorname{dim} D_{x}^{1}=0$, that is, the submanifold $M$ is a Sasakian one.

PROOF: The equation (5.2) means

$$
<A_{\phi W} X-n(X) W, Z>=0
$$

for any $X$ in $D$ and $Z$ and $W$ in $D^{1}$. 
On the other hand, we have from (5.3)

$$
\text { to }(U, V)+A_{F V} U=0
$$

for any vector fields $U$ and $V$ tangent to $M$. From this, we obtain $t \sigma(U, X)=0$ for any vector field $U$ tangent to $M$ and $X$ in $D$. Thus we have

$$
\langle\sigma(U, X), \phi W\rangle=\left\langle A_{\phi W} X, U\right\rangle=-\langle\phi \sigma(U, X), W\rangle=0
$$

that is, $A_{\phi W} X=0$ for any $X$ in $D$ and $W$ in $D^{\perp}$. Substituting this equation into (5.4), we get $\operatorname{dim} D_{x}^{1}=0$.

6. A CONTACT CR-PRODUCT OF A SASAKIAN MANIFOLD I.

In this section, we shall define a contact $C R$-product and give a necessary and sufficient condition that a contact $C R$-submanifold is a contact CR-product.

DEFINITION 6.1: A contact $C R$-submanifold $M$ of a Sasakian manifold $\tilde{M}$ is called a contact $C R$-product if it is locally product of $M^{\perp}$ and $M^{\top}$, where $M^{\top}$ denotes the leaf of the distribution $D$.

THEOREM 6.1: A contact CR-submanifold $M$ of a Sasakian manifold $\tilde{M}$ is a contact $C R$-product if and only if

$$
A_{\phi W} X=n(X) W
$$

for any $Y$ in $D$ and $W$ in $D^{\perp}$.

PROOF: Since (6.1) means

$$
<\sigma(X, Z)-n(X) \phi Z, \phi W>=0
$$

for any $X$ in $D$ and $Z$ and $W$ in $D^{1}$, the leaf $M^{1}$ of $D^{1}$ is totally geodesic in $M$. Furthermore, we have

$$
\langle\sigma(X, \phi Y), \phi Z\rangle=\eta(X)\langle Z, \phi Y>=0
$$

for any $X$ and $Y$ in $D$ and $Z$ in $D^{l}$. So, by virtue of PROPOSITION 3.4, the distribution $D$ is integrable.

Let $M^{\top}$ be the leaf of the distribution $D$, then we have from $(6.1)$

$$
\begin{aligned}
& \left.\left\langle\nabla_{X} Y, Z\right\rangle=\left\langle\tilde{\nabla}_{X}^{Y}, Z\right\rangle=\left\langle\phi \tilde{\nabla}_{X} Y, \phi Z\right\rangle=\left\langle\tilde{\nabla}_{X} \phi Y-\tilde{\nabla}_{X} \phi\right) Y, \phi Z\right\rangle=\langle\sigma(X, \phi Y), \phi Z\rangle \\
& =\left\langle A_{\phi Z} X, Y\right\rangle=0
\end{aligned}
$$

for any $X$ and $Y$ in $D$ and $Z$ in $D^{1}$, that 1s, the leaf $M^{\top}$ of $D$ is totally geodesic in $M$. Thus the submanifold $M$ is a contact $C R$-product. 
Conversely, if the submanifold $M$ of a Sasakian manifold $\tilde{M}$ is a contact CR-product, then we have from (5.2)

$$
A_{\phi W} X-n(X) W \varepsilon D
$$

for any $X$ in $D$ and $W$ in $D^{1}$. So, it is sufficient to prove the following:

$$
A_{\phi W} X-\eta(X) W \in D^{1}
$$

for any $X$ in $D$ and $W$ in $D^{\perp}$. In fact, since the distribution $D$ is totally geodesic in $M$, we have

$$
\begin{aligned}
& <A_{\phi W} X-\eta(X) W, Y>=\langle\sigma(X, Y), \phi W\rangle=-\langle\phi \sigma(X, Y), W\rangle=-\left\langle\phi\left(\tilde{\nabla}_{X} Y-\nabla_{X} Y\right), W\right\rangle \\
& =-\left\langle\phi \tilde{\nabla}_{X}, W\right\rangle=\left\langle\nabla_{X} \phi Y, W\right\rangle=0
\end{aligned}
$$

for any $X$ and $Y$ in $D$ and $W$ in $D^{1}$. This means (6.4). By virtue of (6.3) and (6.4), we have $(6.1)$.

\section{A CONTACT CR-PRODUCT OF A SASAKIAN MANIFOLD II.}

In this section, we shall mainly study the second fundamental form of a contact CR-product.

Let $M$ be a contact $C R$-product of a Sasakian manifold $\tilde{M}$. In $M$, we shall calculate the $\tilde{H}_{B}(X, Z)$ for any unit vectors $X$ in $D$ and $Z$ in $D^{1}$, where $\tilde{H}_{B}(X, Z)$ is defined by

$$
\tilde{H}_{B}(X, Z)=-<\tilde{R}(X, \phi X) Z, \phi Z>
$$

By virtue of $(1.14)$ and $(1.16)$, we get

$$
\begin{aligned}
& <R(\tilde{X}, \phi X) Z, \phi Z>=\left\langle\nabla_{X}^{1} \sigma(\phi X, Z), \phi Z>-\left\langle\nabla_{\phi X}^{1} \sigma(X, Z), \phi Z>-\left\langle A_{\phi Z} \nabla_{X} X, Z\right\rangle\right.\right. \\
& -\left\langle A_{\phi Z} X, \nabla_{X} Z>+<A_{\phi Z} \nabla_{\phi X} X, Z>+<A_{\phi Z} X, \nabla_{\phi X} Z>.\right.
\end{aligned}
$$

Since the leaves $M^{\top}$ and $M^{\perp}$ are both totally geodesic in $M$, we have

$$
\nabla_{U} Y \in D \quad \text { and } \quad \nabla_{U} Z \in D^{1}
$$

for any vector field $U$ tangent to $M, Y$ in $D$ and $Z$ in $D^{1}$. Thus we have from (6.1) and (7.2)

$$
\langle\tilde{R}(X, \phi X) Z, \phi Z\rangle=\left\langle\nabla_{X}^{1} \sigma(\phi X, Z), \phi Z\right\rangle-\left\langle\nabla_{\phi X}^{1} \sigma(X, Z), \phi Z\right\rangle-n\left(\nabla_{X} \phi X\right)+n\left(\nabla_{\phi X} X\right) .
$$

On the other hand, we obtain 


$$
n\left(\nabla_{X} \phi X\right)=\left\langle\nabla_{X} \phi X, \xi\right\rangle=\left\langle\tilde{\nabla}_{X} \phi X, \xi\right\rangle=-1+n(X)^{2} .
$$

So, (7.3) can be written as

$$
\begin{aligned}
& <\tilde{R}(X, \phi X) Z, \phi Z\rangle=\left\langle\nabla_{X}^{1} \sigma(\phi X, Z), \phi Z>-\left\langle\nabla_{\phi X}^{1} \sigma(X, Z), \phi Z\right\rangle+1-n(X)^{2}\right. \\
& +\eta\left(\nabla_{\phi X} X\right) .
\end{aligned}
$$

Next, we have from (6.1)

$$
\langle\sigma(X, W), \phi Z\rangle=\langle\xi, X>\langle Z, W\rangle,
$$

from which

$$
\begin{aligned}
& \langle\sigma(X, Z), \phi Z\rangle=\langle\xi, X>, \\
& \langle\sigma(\phi X, Z), \phi Z\rangle=0 .
\end{aligned}
$$

Covariant differentiation of (7.6) and (7.7) along $\phi X$ and $X$ respectively give us

$$
\begin{aligned}
& \left\langle\nabla_{\phi X}^{1} \sigma(X, Z), \phi Z>=-\left\langle\sigma(X, Z), \nabla_{\phi X}^{1} \phi Z>+\left\langle\nabla_{\phi X} \xi, X>+n\left(\nabla_{\phi X} X\right),\right.\right.\right. \\
& \left\langle\nabla_{X}^{1} \sigma(\phi X, Z), \phi Z>=-\left\langle\sigma(\phi X, Z), \nabla_{X}^{1} \phi Z>.\right.\right.
\end{aligned}
$$

Substituting (7.8) and (7.9) into (7.4), we get

$$
\begin{aligned}
<\tilde{R}(X, \phi X) Z, \phi Z> & =-\left\langle\sigma(X, Z), \nabla_{\phi X}^{1} \phi Z>+\left\langle\sigma(\phi X, \dot{Z}), \nabla_{X}^{1} \phi Z\right\rangle\right. \\
& +2\left(1-\eta(X)^{2}\right) .
\end{aligned}
$$

By virtue of (3.9) and (6.1), we can calculate

$$
<\sigma(\phi X, Z), \nabla_{X}^{1} \phi Z>-<\sigma(X, Z), \nabla_{\phi X}^{1} \phi Z>=-2\|\sigma(X, Z)\|^{2}+2 \eta(X)^{2} .
$$

Thus we have

PROPOSITION 7.1: In a contact CR-product of a Sasakian manifold, we have

$$
\tilde{H}_{B}(X, Z)=2\left(\|\sigma(X, Z)\|^{2}-1\right)
$$

for any unit vectors $X$ in $D$ and $Z$ in $D^{1}$.

Especially, if the ambient manifold $\tilde{M}$ is a Sasakian space form $\tilde{M}(k)$, then we have from (1.15)

$$
\tilde{R}(X, \phi X ; \phi Z, Z)=\frac{k-1}{2}\left(1-\eta(X)^{2}\right) .
$$

Thus we have

PROPOSITION 7.2: In a contact $C R$-product of a Sasakian space form $\tilde{M}(k)$, we have 


$$
\|\sigma(X, Z)\|^{2}=\frac{k+3}{4}-\frac{k-1}{4} \eta(X)^{2}
$$

for any unit vectors $X$ in $D$ and $Z$ in $D^{1}$.

By virtue of PROPOSITION 3.4. and $(7.14)$, we have

$$
\begin{aligned}
& \|\sigma(X, Z)\|^{2}=\frac{k+3}{4} \quad \text { for } X \perp \xi, \\
& \|\sigma(\xi, Z)\|^{2}=1
\end{aligned}
$$

Thus we have

COROLLARY 7.3: In a Sasakian space form $\tilde{M}(k)$ with constant $\phi$-holomorphic sectional curvature $k<-3$, there does not exist a contact $C R$-product of $\tilde{M}(k)$.

Next, we shall prove

THEOREM 7.4: Let $M$ be a contact $C R$-submanifold of a Sasakian space form $\tilde{M}(k)$. Then we have

$$
\|\sigma\|^{2} \geqq 2 p\left(\frac{h(k+3)}{2}+1\right)
$$

where $p=\operatorname{dim} D^{1}$ and $2 h=\operatorname{dim} D-1$. If the equality sign of (7.16) holds, then $M^{\top}$ and $M^{1}$ are both totally geodesic in $\tilde{M}(k)$.

PROOF: Let $A_{1}, A_{2}, \ldots, A_{h}, \phi A_{1}, \phi A_{2}, \ldots, \phi A_{h}, A_{2 h+1}(=\xi)$ and $B_{1}, B_{2}, \ldots, B_{p}$ be orthogonal basis of $D_{x}$ and $D_{x}^{1}$, respectively. Then $\|\sigma\|^{2}$ is given by

$$
\|\sigma\|^{2}=\sum_{i, j=1}^{2 h+1}\left\|\sigma\left(A_{i}, A_{j}\right)\right\|^{2}+2 \sum_{i=1}^{2 h+1} \sum_{\alpha=1}^{p}\left\|\sigma\left(A_{i}, B_{\alpha}\right)\right\|^{2}+\sum_{\alpha, \beta=1}^{p}\left\|\sigma\left(B_{\alpha}, B_{\beta}\right)\right\|^{2} .
$$

By virtue of (7.15), the above equation can be written as

$$
\left.\|\sigma\|^{2}=2 p\left(\frac{h(k+3)}{2}\right)+1\right)+\sum_{i, j=1}^{2 h+1}\left\|\sigma\left(A_{i}, A_{j}\right)\right\|^{2}+\sum_{\alpha, \beta=1}^{p}\left\|\sigma\left(B_{\alpha}, B_{\beta}\right)\right\|^{2} .
$$

From the above equation, we have our theorem.

\section{TOTALLY UMBILICAL CONTACT CR-SUBMANIFOLDS.}

Let $M$ be a totally umbilical contact $C R$-submanifold of a Sasakian manifold $\tilde{M}$. Then by definition we have

$$
\sigma(U, V)=\langle U, V\rangle
$$

for any vector fields $U$ and $V$ tangent to $M$. By virtue of (1.6), we can write

$$
\phi H=t H+f H .
$$

Since the vector field $t H$ is in $D^{1}$, we have from (3.8) 


$$
A_{\phi t H^{W}}=A_{\phi W} t H
$$

for any $W$ in $D^{\perp}$. From this, we obtain

$$
-\langle W, W<t H, t H>=\langle t H, W>\langle H, \phi W>
$$

We assume that $\operatorname{dim} D^{\perp} \geqq 2$. Then we can put $W$ as the orthogonal vector field of $t H$. The equation ( 8.4$)$ means

$$
t H=0
$$

Next, let $Q_{1}$ and $Q_{2}$ be the projections of $T M$ to $D$ and $D^{1}$, respectively. Then for any vector field $U$ tangent to $M$ we can put

$$
U=Q_{1} U+Q_{2} U
$$

and

$$
\phi Q_{1} U \in D, \quad \phi Q_{2} U \in \phi D^{1} \subset T^{1} M .
$$

The equation (8.7) and the covariant differentiation of $\phi \lambda=t \lambda+f \lambda$ teach us

$$
-\phi Q_{1} A_{\lambda} U=Q_{1} \nabla_{U} t \lambda-Q_{1} A_{f \lambda} U
$$

for any vector field $U$ tangent to $M$ and any vector field $\lambda$ normal to $M$. In (8.8), if we put $\lambda=H$ and taking account of (8.5), we have

$$
\phi Q_{1} A_{H} U=Q_{1} A_{\phi H^{U}}
$$

for any vector field $U$ tangent to $M$. For any $X$ in $D$ and any vector field $U$ tangent to $M$, we get

$$
\begin{aligned}
& \left\langle Q_{1} A_{\phi H} U, X>=\left\langle A_{\phi H} U, X\right\rangle=\langle\sigma(U, X), \phi H\rangle=\langle U, X>\langle H, \phi H\rangle=0,\right. \\
& \left\langle\phi Q_{1} A_{H} U, X>=-\left\langle Q_{1} A_{H} U, \phi X>=-\left\langle A_{H} U, \phi X\right\rangle=-\langle\sigma(U, \phi X), H\rangle=-\langle U, \phi X\rangle\langle H, H\rangle .\right.\right.
\end{aligned}
$$

By virtue of (8.9) and the above two equations, we have

$$
\langle H, H\rangle\langle U, \phi X>=0
$$

for any vector field $U$ tangent to $M$ and any $X$ in $D$. We assume that $\operatorname{dim} D_{x} \geqq 2$ and if we take $U=\phi X$ such that the vector field $X$ is orthogonal to $\xi$, we have from (8.10) $H=0$. Thus we have

THEOREM 8.1: Let $M$ be a totally umbilical contact $C R$-submanifold of a Sasakian 
manifold $\tilde{M}$. We assume that $\operatorname{dim} D_{x} \geqq 2$ and $\operatorname{dim} D_{x}^{1} \geqq 2$. Then the submanifold $M$ is totally geodesic in $\tilde{M}$.

\section{REFERENCES}

1. Bejancu, A. CR-submanifolds of a Kaehler manifold I, Proc. Amer. Math. Soc.,69 (1978) 135-142.

2. Chen, B. Y. On CR-submanifolds of a Kaehler manifold I, J. Differential Geometry, 16 (1981) 305-322.

3. Ogiue, K. On almost contact manifolds admitting axiom of planes or axiom of free mobility, Kodai Math. Sem. Rep., 16(1964) 223-232.

4. Sasaki, S. On differential manifolds with $(\Phi, \Psi)$-structures, Tôhoku Math. J. 13 (1961) 132-153.

5. Yano, K. and Kon, M. Differential geometry of CR-submanifolds, Geometria Dedicata, 10(1981) 369-391. 


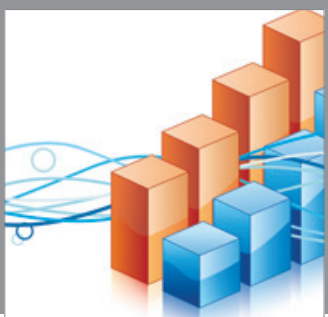

Advances in

Operations Research

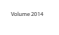

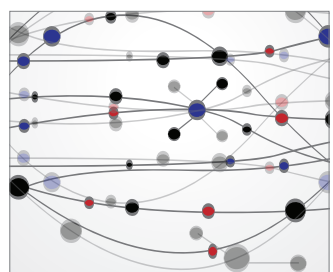

\section{The Scientific} World Journal
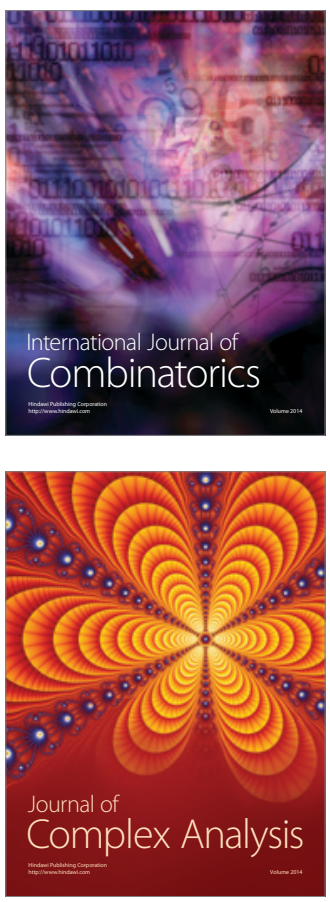

International Journal of

Mathematics and

Mathematical

Sciences
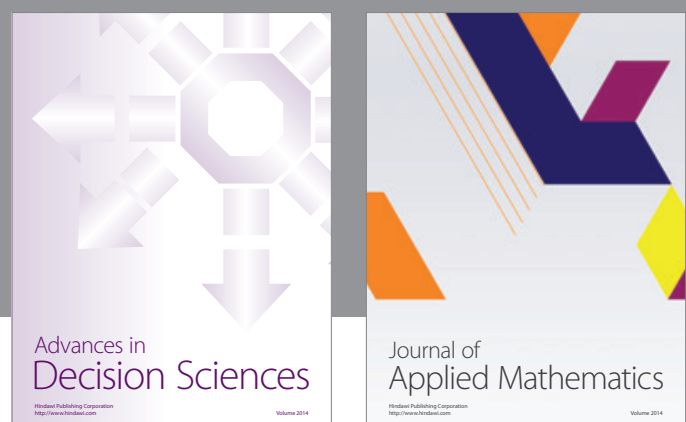

Journal of

Applied Mathematics
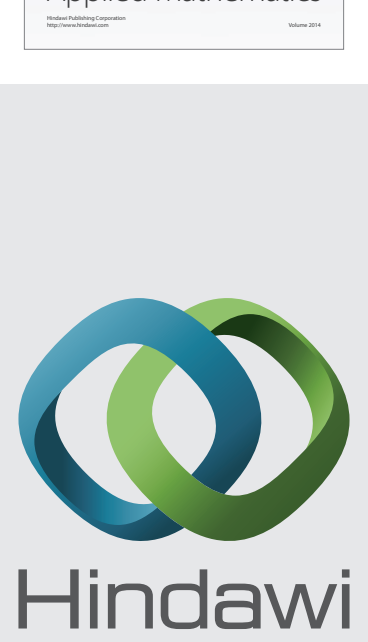

Submit your manuscripts at http://www.hindawi.com
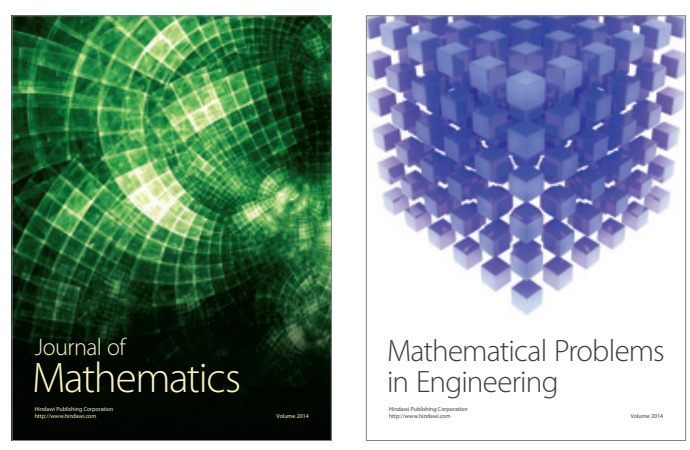

Mathematical Problems in Engineering
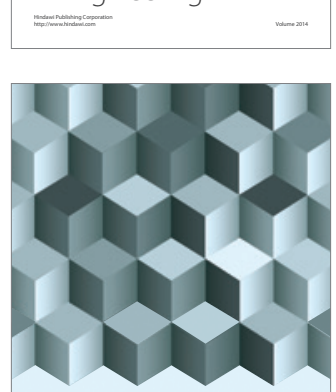

Journal of

Function Spaces
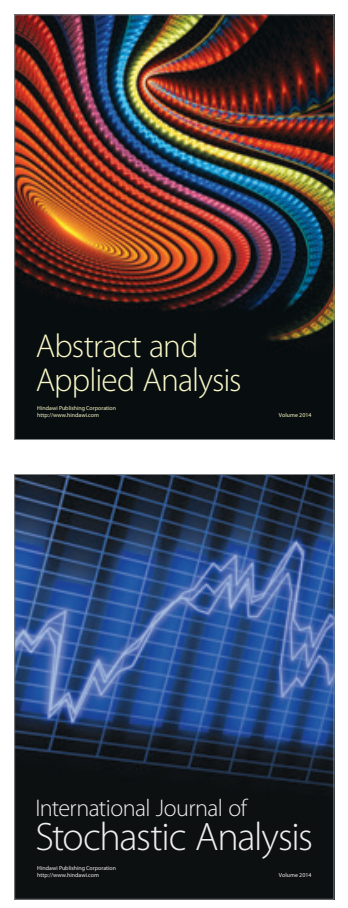

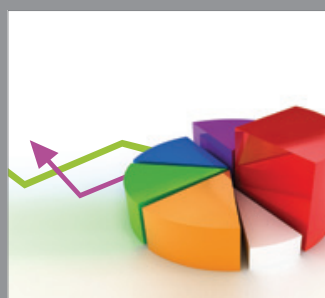

ournal of

Probability and Statistics

Promensencen
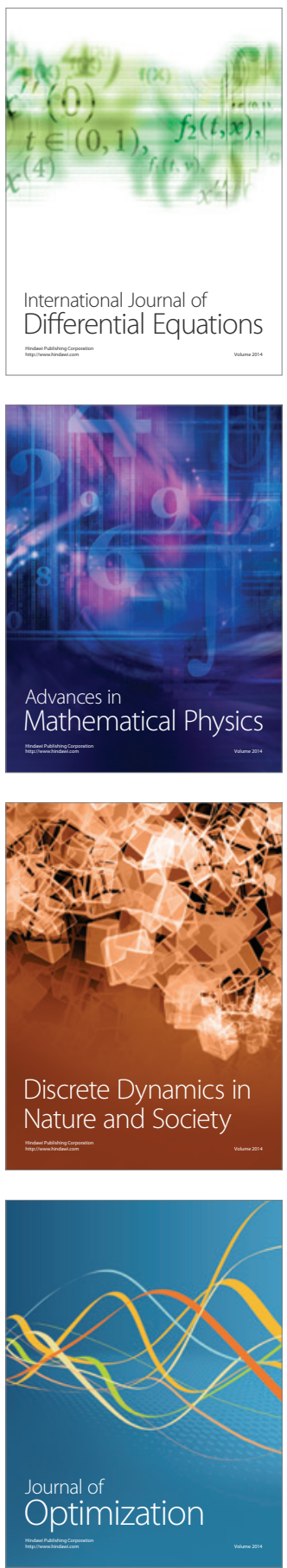